Pacific Journal of Mathematics

SHAPE GROUPS AND PRODUCTS 


\section{SHAPE GROUPS AND PRODUCTS}

\section{Thomas J. SANDERS}

In recent papers $S$. Mardešić and J. Segal have used ANR-systems to obtain an alternate approach to Borsuk's shape theory. At the same time, they have extended the theory to include all compact Hausdorff spaces. In this paper some of the results of Mardešic and Segal are used to obtain Borsuk's fundamental groups, and to extend some of Borsuk's results that relate shapes and products to arbitrary products. A result relating (direct) products and shape groups is also obtained.

The reader is referred to Chapter 1 of [11] for all categorical definitions; e.g., inverse system, terminal object, inverse limit.

If $X=\left\{X_{a}, p_{a a^{\prime}}, A\right\}$ and $Y=\left\{Y_{b}, q_{b b^{\prime}}, B\right\}$ are inverse systems in a category $\mathscr{A}$, a morphism of inverse systems $\underline{f}: \underline{X} \rightarrow \underline{Y}$ consists of an increasing function $f: B \rightarrow A$ and a collection of $\mathscr{A}$-morphisms $f_{b}: X_{f(b)} \rightarrow$ $Y_{b}$ such that if $b \leqq b^{\prime}$ then $f_{b} p_{f(b) f\left(b^{\prime}\right)}=q_{b b^{\prime}} f_{b^{\prime}}$. If $X_{\infty}=\lim \underline{X}$ and $Y_{\infty}=\lim \underline{Y}$ exist and $\underline{f}: \underline{X} \rightarrow \underline{Y}$ is a morphism of inverse systems then for each $b \in B$ the composition $f_{b} p_{f(b)}: X_{\infty} \rightarrow Y_{b}$ satisfies if $b \leqq b^{\prime}$ then

$$
f_{b} p_{f(b)}=f_{b} p_{f(b) f\left(b^{\prime}\right)} p_{f\left(b^{\prime}\right)}=q_{b b^{\prime}} f_{b^{\prime}} p_{f\left(b^{\prime}\right)} .
$$

By the universal mapping property of $Y_{\infty}$ there is a unique $\mathscr{A}$-morphism $f_{\infty}: X_{\infty} \rightarrow Y_{\infty}$ such that if $b \in B$ then $q_{b} f_{\infty}=f_{b} p_{f(b)}$. The $\mathscr{A}$-morphism $f_{\infty}$ is said to be induced by $\underline{f}$.

Under the usual definitions of composition and identities, there is a category, denoted inv $(\mathscr{A})$, whose objects are inverse systems in $\mathscr{A}$ and whose morphisms are morphisms of inverse systems. The reader will note that this category is not the category used by Mardešic and Segal [6]. It is not difficult to show that if $\underline{f}: \underline{X} \rightarrow \underline{Y}$ and $\underline{g}: \underline{Y} \rightarrow \underline{Z}$ are morphisms of inverse systems and if $X_{\infty}, Y_{\infty}$ and $Z_{\infty}=$ $\lim \underline{Z}$ exist then $(g f)_{\infty}=g_{\infty} f_{\infty}$.

An $A N R$-system is an inverse system $X=\left\{\left(X_{a}, x_{a}\right), P_{a a^{\prime}}, A\right\}$ in the category of pointed topological spaces where $A$ is closure-finite (i.e., for every $a \in A$, the set of predecessors of $a$ is finite) and each $X_{a}$ is a compact ANR for normal spaces. This definition differs from that usually given in that the authors in [6] required each $X_{a}$ to be compact ANR for metric spaces. This condition is easily relaxed; as was done in [5]. As in [5], we use notions and results from [6] in this setting without specific citations. 
If $X$ is a compact Hausdorff space, $x_{0} \in X \subset M$, an inclusion $A N R$ system in $M$ associated with $\left(X, x_{0}\right)$ is an ANR-system $\underline{X}=\left\{\left(X_{a}, x_{0}\right)\right.$, $\left.i_{a a^{\prime}}, A\right\}$ associated with $\left(X, x_{0}\right)$ where

(1) each $X_{a}$ is a neighborhood of $X$ in $M$

(2) $X=\bigcap_{a \in A} X_{a}$

(3) if $a \leqq a^{\prime}$ then $i_{a a^{\prime}}:\left(X_{a^{\prime}}, x_{0}\right) \rightarrow\left(X_{a}, x_{0}\right)$ is an inclusion map. If $A=N$ (the set of natural numbers) then $X$ is said to be an inclusion $A N R$-sequence and is denoted $X=\left\{\left(X_{k}, x_{0}\right), i_{k k^{\prime}}\right\}$. If $X$ is contained in a parallelotope $I^{\Omega}=\prod_{w \in \Omega} I_{\omega}, I_{\omega}=I$, then [5] $\left(X, x_{0}\right)$ has an associated inclusion ANR-system (sequence if $\Omega$ is countable).

Another useful category is the category of ANR-systems, developed by Mardešic and Segal in [6]. The objects of this category are ANRsystems $X=\left\{\left(X_{a}, x_{a}\right), P_{a a^{\prime}}, A\right\}$ (recall our definition differs somewhat from that used in [6]). A morphism in this category $\underline{f}: \underline{X} \rightarrow \underline{Y}=$ $\left\{\left(Y_{b}, y_{b}\right), q_{b b^{\prime}}, B\right\}$, called a map of systems, consists of an increasing function $f: B \rightarrow A$ and a collection of maps (i.e., continuous functions) $f_{b}: X_{f(b)} \rightarrow Y_{b}$ such that if $b \leqq b^{\prime}$ then $f_{b} P_{f(b) f\left(b^{\prime}\right)} \cong q_{b b^{\prime}} f_{b^{\prime}}$; i. e., the diagram

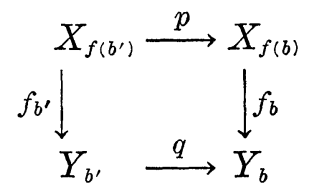

commutes up to homotopy.

2. The shape groups. In [6], Mardešić and Segal define the concept of homotopy between two maps of systems. To be more precise, two maps of systems $\underline{f}, \underline{g}: \underline{X} \rightarrow \underline{Y}$ are said to be homotopic, written $\underline{f} \cong \underline{g}$, provided that for every $b \in B$ there is an $a \in A, a \geqq f(b), g(b)$, such that $f_{b} p_{f(b) a} \cong g_{b} p_{g(b) a}$. Noting the similarities between the category inv $(\mathscr{A})$ and the category of ANR-systems one can define a similar relation in inv ( $\mathscr{A})$.

Let $\mathscr{A}$ be a category. Two morphisms $\underline{f}, \underline{g}: \underline{X} \rightarrow \underline{Y}$ of inverse systems in $\mathscr{A}$ are $\sim$-related $(\underline{f} \sim \underline{g})$ if for each $b \in B$ there is an index $a \in A, a \geqq f(b), g(b)$ such that $f_{b} p_{f(b) a}=g_{b} p_{g(b) a}$.

THEOREM 2.1. The relation $\sim$ is an equivalence relation.

Proof. The proof is as in Theorem 2 of [6].

Theorem 2.2. Let $\underline{f}, \underline{f}^{\prime}: \underline{X} \rightarrow \underline{Y}$ and $\underline{g}, \underline{g^{\prime}}: \underline{Y} \rightarrow \underline{Z}$. If $f \sim f^{\prime}$ and $\underline{g} \sim \underline{g}^{\prime}$ then $\underline{g f} \sim \underline{g}^{\prime} \underline{f}^{\prime}$. 


\section{Proof. See Theorem 3 of [6].}

A morphism $f: \underline{X} \rightarrow \underline{Y}$ is a $\sim$-equivalence provided there is a morphism $\underline{g}: \underline{Y} \rightarrow \underline{X}$ (called the $\sim$-inverse of $\underline{f}$ ) such that $\underline{g f} \sim \underline{1}_{\underline{X}}$ and $\underline{f g} \sim \underline{1}_{\underline{Y}}$. In this case, $\underline{X}$ and $\underline{Y}$ are said to be $\sim$-equivalent $(\underline{X} \sim \underline{Y})$.

THEOREM 2.3. The relation $\sim$ is an equivalence relation of inverse systems in $\mathscr{A}$.

Proof. See Theorem 4 of [6].

THEOREM 2.4. If $\underline{f}, \underline{g}: \underline{X} \rightarrow \underline{Y}$ are $\sim$-related morphisms and $X_{\infty}$ and $Y_{\infty}$ both exist then $f_{\infty}=g_{\infty}$.

Proof. By definition, $f_{\infty}: X_{\infty} \rightarrow Y_{\infty}$ is the unique $\mathscr{A}$-morphism satisfying for all $b \in B, q_{b} f_{\infty}=f_{b} p_{f(b)}$. Similarily, $g_{\infty}: X_{\infty} \rightarrow Y_{\infty}$ is the unique $\mathscr{A}$-morphism satisfying for all $b \in B, q_{b} g_{\infty}=g_{b} p_{g(b)}$. Choose $a \geqq f(b)$, $g(b)$ such that $f_{b} p_{f(b) a}=g_{b} p_{g(b) a}$. Now $p_{f(b)}=p_{f(b) a} p_{a}$ and $p_{g(b)}=p_{g(b) a} p_{a}$ so that

$$
q_{b} g_{\infty}=g_{b} p_{g(b)}=g_{b} p_{g(b) a} p_{a}=f_{b} p_{f(b) a} p_{a}=f_{b} p_{f(b)} .
$$

By the uniqueness, $f_{\infty}=g_{\infty}$.

CoROLlary 2.5. If $\underline{X} \sim \underline{Y}$ and $X_{\infty}, Y_{\infty}$ both exist then $X_{\infty}$ and $Y_{\infty}$ are $\mathscr{C}$-equivalent objects.

Proof. If $\underline{f}: \underline{X} \rightarrow \underline{Y}$ and $\underline{g}: \underline{Y} \rightarrow \underline{X}$ are such that $\underline{g f} \sim \underline{1}_{\underline{X}}$ and $\underline{f g} \sim \underline{1}_{\underline{Y}}$ then $g_{\infty} f_{\infty}=(g f)_{\infty}=1_{X_{\infty}}$ and $f_{\infty} g_{\infty}=(f g)_{\infty}=1_{Y_{\infty}}$.

If $X=\left\{\left(X_{a}, x_{a}\right), p_{a a^{\prime}}, A\right\}$ is an ANR-system, let $\pi_{n}(\underline{X})=\left\{\left(\pi_{n}\left(X_{a}, 2_{a}\right)\right.\right.$, $\left.\rho_{a a^{\prime}}, A\right\}$ denote the inverse system of groups where $\pi_{n}\left(X_{a}, x_{a}\right)$ is the $n$th homotopy group of $\left(X_{a}, x_{a}\right)$ and if $a \leqq \alpha^{\prime}$ then $\rho_{a a^{\prime}}: \pi_{n}\left(X_{a^{\prime}}, x_{a^{\prime}}\right) \rightarrow \pi_{n}\left(X_{a}, x_{a}\right)$ is the homomorphism induced by $p_{a a^{\prime}}$; i.e., if $[\xi] \in \pi_{n}\left(X_{a^{\prime}}, x_{a^{\prime}}\right)$ then $\rho_{a a},[\xi]=\left[p_{a a}, \xi\right]$.

If $\underline{f}: \underline{X} \rightarrow \underline{Y}$ is a map of systems, $f$ induces a morphism of inverse systems $\underline{f}_{*}: \pi_{n}(\underline{X}) \rightarrow \pi_{n}(\underline{Y})$ where $f_{*}=f: B \rightarrow A$ and $\left(f_{b}\right)_{*}: \pi_{n}\left(X_{f(b)}\right.$, $\left.x_{f(b)}\right) \rightarrow \pi_{n}\left(Y_{b}, y_{b}\right)$ is the homomorphism induced by $f_{b}$. This gives a covariant functor $\pi_{n}$ between the category of ANR-systems and the category of inverse systems of groups.

THEOREM 2.6. If $\underline{f}, \underline{g}: \underline{X} \rightarrow \underline{Y}$ are homotopic maps of systems $(\underline{f} \cong$ $\underline{g})$ then the induced morphisms $\underline{f}_{*}, \underline{g}_{*}: \pi_{n}(\underline{X}) \rightarrow \pi_{n}(\underline{Y})$ are $\sim$-related $\left(\underline{f}_{*} \sim \underline{g}_{*}\right)$. 
Proof. For each $b \in B$, choose $a \in A$ such that $a \geqq f(b), g(b)$ and $f_{b} p_{f(b) a} \cong g_{b} p_{g(b) a}$. Then if $[\xi] \in \pi_{n}\left(X_{a}, x_{a}\right)$,

$$
\left(f_{b}\right)_{*} \rho_{f(b) a}[\xi]=\left[f_{b} p_{f(b) a} \xi\right]=\left[g_{b} p_{g(b) a} \xi\right]=\left(g_{b}\right)_{*} \rho_{g(b) a}[\xi] \text {. }
$$

CoROLlaRY 2.7. If $\underline{X} \cong \underline{Y}$ then $\pi_{n}(\underline{X}) \sim \pi_{n}(\underline{Y})$.

Mardeśic and Segal have shown in [6] that if $\underline{X}$ and $\underline{Y}$ are ANRsystems associated with $\left(X, x_{0}\right)$ and $\left(Y, y_{0}\right)$, respectively, then a map $f:\left(X, x_{0}\right) \rightarrow\left(Y, y_{0}\right)$ has an associated map of systems $\underline{f}: \underline{X} \rightarrow \underline{Y}$. If $\underline{X}$ and $\underline{X}^{\prime}$ are ANR-systems associated with $\left(X, x_{0}\right)$ then any map of systems $\underline{i}: \underline{X} \rightarrow \underline{X}^{\prime}$ associated with the identity $1_{X, x_{0}}:\left(X, x_{0}\right) \rightarrow\left(X, x_{0}\right)$ is a homotopy equivalence. By Corollary 2.7 and Corollary 2.5, $i_{\infty}: \lim \pi_{n}(\underline{X}) \rightarrow$ $\lim \pi_{n}\left(\underline{X}^{\prime}\right)$ is an isomorphism. Suppose $f:\left(X, x_{0}\right) \rightarrow\left(Y, y_{0}\right)$ is a map,

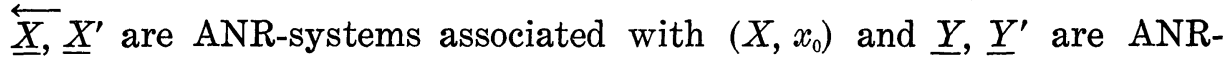
systems associated with $\left(Y, y_{0}\right)$. Let $\underline{i}: \underline{X} \rightarrow \underline{X}^{\prime}$ and $\underline{j}: \underline{Y} \rightarrow \underline{Y}^{\prime}$ be homotopy equivalences associated with $1_{X, x_{0}}$ and $1_{Y, y_{0}}$, respectively. Let $\underline{f}: \underline{X} \rightarrow \underline{Y}$ and $\underline{f}^{\prime}: \underline{X}^{\prime} \rightarrow \underline{Y}^{\prime}$ be maps of systems associated with $f$. It follows [5] that $\underline{j f} \cong \underline{f}^{\prime} \underline{i}: \underline{X} \rightarrow \underline{Y}^{\prime}$. By Corollary 2.7 and Corollary 2.5, one has that $j_{\infty} f_{\infty}=f_{\infty}^{\prime} i_{\infty}: \lim \pi_{n}(\underline{X}) \rightarrow \lim \pi_{n}\left(\underline{Y}^{\prime}\right)$.

If $\left(X, x_{0}\right)$ is a pointed compact Hausdorff space and $\underline{X}$ is any ANR-system associated with $\left(X, x_{0}\right)$ then the nth shape group ${ }^{1}$ of $\left(X, x_{0}\right)$ is given by $\underline{\pi}_{n}\left(X, x_{0}\right)=\lim \pi_{n}(\underline{X})$. If $f:\left(X, x_{0}\right) \rightarrow\left(Y, y_{0}\right)$ then the homomorphism $f_{\infty}: \underline{\pi}_{n}\left(X, x_{0}\right) \leftrightarrows \underline{\pi}_{n}\left(Y, y_{0}\right)$ is said to be induced by $f$. It is easy to show that $\left(1_{X, x_{0}}\right)_{\infty}=1_{\underline{n}_{n}\left(X, x_{0}\right)}$ and $(f g)_{\infty}=f_{\infty} g_{\infty}$. Corollary 2.7 also shows that the $n$th shape group is a shape invariant. It is shown in $\S 3$ that this definition of $\underline{\pi}_{n}$ extends that given by Borsuk in [1].

THEOREM 2.8. There is a homomorphism $p: \pi_{n}\left(X, x_{0}\right) \rightarrow \underline{\pi}_{n}\left(X, x_{0}\right)$ such that for all $a \in A,\left(p_{a}\right)_{*}=\rho_{a} p$ where $\left(p_{a}\right)_{*}: \pi_{n}\left(X, x_{0}\right) \rightarrow \pi_{n}\left(X_{a}, x_{a}\right)$ is the homomorphism induced by $p_{a}:\left(X, x_{0}\right) \rightarrow\left(X_{a}, x_{a}\right)$.

Proof. The collection of maps $p_{a}:\left(X, x_{0}\right) \rightarrow\left(X_{a}, x_{a}\right)$ induces homomorphisms $\left(p_{a}\right)_{*}: \pi_{n}\left(X, x_{0}\right) \rightarrow \pi_{n}\left(X_{a}, x_{a}\right)$ such that if $a \leqq a^{\prime}$ then $\left(p_{a}\right)_{*}=$ $\rho_{a a^{\prime}}\left(p_{a^{\prime}}\right)_{*}$. By the universal mapping property of $\pi_{n}\left(X, x_{0}\right)$ there is a unique homomorphism $p: \pi_{n}\left(X, x_{0}\right) \rightarrow \underline{\pi}_{n}\left(X, x_{0}\right)$ such that for all $a \in A$, $\left(p_{a}\right)_{*}=\rho_{a} p$.

Theorem 2.9. If $X \in A N R$ then $\pi_{n}\left(X, x_{0}\right) \approx \underline{\pi}_{n}\left(X, x_{0}\right)$.

Proof. Since $X \in \mathrm{ANR}$, there is a special ANR-system $\underline{X}=$

\footnotetext{
1 Note the $n$th shape group is actually an isomorphism class of groups.
} 
$\left\{\left(X, x_{0}\right), 1_{X, x_{0}}\right\}$ associated with $\left(X, x_{0}\right)$. Then $\pi_{n}(\underline{X})=\left\{\pi_{n}\left(X, x_{0}\right), 1_{\pi_{n}\left(X, x_{0}\right.}\right\}$ has as inverse limit the group $\pi_{n}\left(X, x_{0}\right)$.

THEOREM 2.10. If $X$ is a compact Hausdorff space, $x_{0} \in X$ and $X_{0}$ is the component of $X$ containing $x_{0}$ then $\underline{\pi}_{n}\left(X, x_{0}\right)=\underline{\pi}_{n}\left(X_{0}, x_{0}\right)$.

Proof. Assume $X \subset I^{\Omega}$ and $\underline{X}=\left\{\left(X_{a}, x_{0}\right), i_{a a^{\prime}}, A\right\}$ is an inclusion ANR-system associated with $\left(X, x_{0}\right)$. For each $a \in A$ let $X_{a 0}$ denote the component of $X_{a}$ containing $x_{0}$. Since a compact ANR is locally contractible, it is locally path connected. It follows that each $X_{a 0}$ is a compact path connected ANR.

Claim. $\underline{X}_{0}=\left\{\left(X_{a 0}, x_{0}\right),\left.i_{a a^{\prime}}\right|_{X_{a^{\prime} 0}}, A\right\}$ is an inclusion ANR-system associated with $\left(X_{0}, x_{0}\right)$. It suffices to show that $X_{0}=\bigcap_{a \in A} X_{a 0}$. Certainly $X_{0} \subset \bigcap_{a \in A} X_{a 0}$ since $X_{0}$ a compact connected subset of $I^{a}$ implies that if $N$ is any neighborhood of $X_{0}$ there is a path connected neighborhood $U$ of $X_{0}$ such that $U \subset N$. Let $x \in \bigcap_{a \in A} X_{a 0}-X_{0}$. Then $x \in X-X_{0}$ so let $X_{1}$ denote the component of $X$ to which $x$ belongs. Then there are disjoint open sets $U_{0}, U_{1}$ such that $U_{i} \cap X=X_{i}(i=0,1)$. Since $I^{2}$ is normal, there are open sets $V_{0}, V_{1}$ such that $X_{i} \subset V_{i} \subset \bar{V}_{i} \subset U_{i}(i=0,1)$. Since $V=V_{0} \cup V_{1} \cup\left[I^{\Omega}-\left(\bar{V}_{0} \cup \bar{V}_{1}\right)\right]$ is a neighborhood of $X$ in $I^{\Omega}$ there is an $a \in A$ such that $X_{a} \subset V$. Now $X_{a 0} \subset V_{0}$ and $x \in V_{1}$ a contradiction since $V_{0} \cap V_{1}=\varnothing$. Thus $X_{0}=\bigcap_{a \in A} X_{a 0}$ and the claim is proven. By a well-known theorem, $\pi_{n}\left(X_{a}, x_{0}\right)=\pi_{n}\left(X_{a 0}, x_{0}\right)$ so that $\pi_{n}(\underline{X})=\pi_{n}\left(\underline{X}_{0}\right)$. It follows then that $\underline{\pi}_{n}\left(X, x_{0}\right)=\underline{\pi}_{n}\left(X_{0}, x_{0}\right)$.

If $x_{0}, x_{1} \in X$ and $\omega: I \rightarrow X$ is a path in $X$ connecting $x_{0}$ and $x_{1}$ then for each $a \in A, \omega$ induces an isomorphism $\omega_{a}: \pi_{n}\left(X_{a}, x_{0}\right) \rightarrow \pi_{n}\left(X_{a}, x_{1}\right)$. If $a \leqq a^{\prime}$ then $i_{a a^{\prime}} \omega_{a^{\prime}}=\omega_{a}$ and it is not hard to show that $\underline{\pi}_{n}\left(X, x_{0}\right) \approx$ $\underline{\pi}_{n}\left(X, x_{1}\right)$. Thus we have the following theorem.

THEOREM 2.11. If $x_{0}$ and $x_{1}$ are in the same path component of $X$ then $\underline{\pi}_{a}\left(X, x_{0}\right) \approx \underline{\pi}_{n}\left(X, x_{1}\right)$.

Question. Is Theorem 2.11 valid if one replaces path component with component? Using Theorem 4.1 of [4], one can easily show the following is true.

THEOREM 2.12. If $X$ is a movable compact metric space and if $x_{0}$ and $x_{1}$ are in the same component of $X$ then $\underline{\pi}_{n}\left(X, x_{0}\right) \approx \underline{\pi}_{n}\left(X, x_{1}\right)$.

3. Equivalence of the inverse limit and Borsuk's definition of $\underline{\pi}_{n}$. Let $X$ be a compact metric space and $x_{0} \in X$. Assume that $X$ is embedded in $Q$ (Hilbert cube). Let $(S, a)$ denote the pointed $n$ dimensional sphere. An approximative map of $(S, a)$ toward $\left(X, x_{0}\right)$, 
$\xi=\left\{\xi_{k},(S, a) \rightarrow\left(X, x_{0}\right)\right\}$ is a sequence of maps $\xi_{k}:(S, a) \rightarrow\left(Q, x_{0}\right)$ such that for every neighborhood $\left(V, x_{0}\right)$ of $\left(X, x_{0}\right)$ in $Q$ there is an index $k_{0}$ such that if $k \geqq k_{0}$ then $\xi_{k} \cong \xi_{k+1}$ in $\left(V, x_{0}\right)$. Two approximative maps $\underline{\xi}$ and $\underline{\eta}=\left\{\eta_{k},(S, a) \rightarrow\left(X, x_{0}\right)\right\}$ are said to be homotopic, $\underline{\xi} \cong \underline{\eta}$, if for each neighborhood $\left(V, x_{0}\right)$ of $\left(X, x_{0}\right)$ in $Q$ there is an index $\bar{k}_{0}$ such that if $k \geqq k_{0}$ then $\xi_{k} \cong \eta_{k}$ in $\left(V, x_{0}\right)$. The homotopy class of an approximative map $\underline{\xi}$ is denoted by $[\xi]$.

If $\xi, \eta:(S, a) \rightarrow\left(Q, x_{0}\right)$ are maps, their join, $\xi * \eta:(S, a) \rightarrow\left(Q, x_{0}\right)$ is defined as follows. Let $P$ and $P^{\prime}$ be $n$-dimensional balls on $S$ such that $a \in S-\dot{P}, a \in S-\dot{P}^{\prime}$ and $\dot{P}^{\prime} \subset S-\dot{P}$. Let $\alpha, \beta:(S, a) \times I \rightarrow(S, a)$ be homotopies such that $\alpha(x, 0)=\beta(x, 0)=x$ for all $x \in S$ and $\alpha(S-$ $\stackrel{\circ}{P}, 1)=a=\beta\left(S-\stackrel{\circ}{P}^{\prime}, 1\right)$. Define

$$
(\xi * \eta)(x)= \begin{cases}\xi \alpha(x, 1) & \text { if } x \in S-\dot{P}^{\prime} \\ \eta \beta(x, 1) & \text { if } x \in S-\dot{P} .\end{cases}
$$

Note: if $[\xi],[\eta] \in \pi_{n}\left(X, x_{0}\right)$ where $\left(X, x_{0}\right) \subset\left(Q, x_{0}\right)$ then $[\xi] *[\eta]=[\xi * \eta]$ is the group operation in $\pi_{n}\left(X, x_{0}\right)$. Let $\underline{\pi}_{n}^{Q}\left(X, x_{0}\right)$ denote the group of fundamental classes of approximative maps of $(S, a)$ toward $\left(X, x_{0}\right)$ with operation $*$ defined as follows. If $[\xi],[\underline{\eta}] \in \underline{\pi}_{n}^{Q}\left(X, x_{0}\right)$ then

$$
[\underline{\xi}] *[\underline{\eta}]=\left[\left\{\xi_{k} * \eta_{k},(S, a) \longrightarrow\left(X, x_{0}\right)\right\}\right] \text {. }
$$

Then [3] $\underline{\pi}_{n}^{Q}\left(X, x_{0}\right)$ is the $n$th fundamental group defined by Borsuk in [1].

THEOREM 3.1. If $\underline{X}=\left\{\left(X_{k}, x_{0}\right), i_{k k^{\prime}}\right\}$ is an inclusion ANR-sequence in $Q$ associated with $\left(X, x_{0}\right) \subset\left(Q, x_{0}\right)$ then $\underline{\pi}_{n}^{Q}\left(X, x_{0}\right) \approx \lim \pi_{n}(\underline{X})=$ $\underline{\pi}_{n}\left(X, x_{0}\right)$.

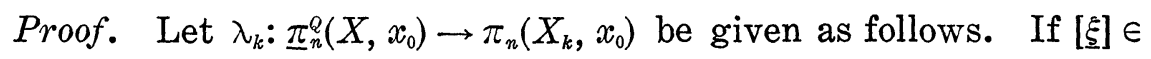
$\underline{\pi}_{n}^{Q}\left(X, x_{0}\right)$ then since $\left(X_{k}, x_{0}\right)$ is a neighborhood of $\left(X, x_{0}\right)$ in $Q$ there is an index $m_{k}$ such that if $m \geqq m_{k}$ then $\xi_{m} \cong \xi_{m_{k}}$ in $\left(X_{k}, x_{0}\right)$. Define $\lambda_{k}[\underline{\xi}]=\left[\xi_{m_{k}}\right] \in \pi_{n}\left(X_{k}, x_{0}\right)$. If $[\underline{\xi}]=[\underline{\eta}]$ then there is an $m_{0}$ such that if $m \geqq m_{0}$ then $\xi_{m} \cong \eta_{m}$ in $\left(X_{k}, x_{0}\right)$ so that $\lambda_{k}$ is a well-defined function. If $[\xi],[\eta]] \in \underline{\pi}_{n}^{Q}\left(X, x_{0}\right)$ and $m_{0}$ is "large enough" then

$$
\begin{aligned}
\lambda_{k}([\underline{\xi}] *[\underline{\eta}]) & =\lambda_{k}\left[\left\{\xi_{m} * \eta_{m},(S, a) \longrightarrow\left(X, x_{0}\right)\right\}\right] \\
& =\left[\xi_{m_{0}} * \eta_{m_{0}}\right] \\
& =\left[\xi_{m_{0}}\right] *\left[\eta_{m_{0}}\right] \\
& =\lambda_{k}[\underline{\xi}] * \lambda_{k}[\underline{\eta}] .
\end{aligned}
$$

Thus each $\lambda_{k}$ is a group homomorphism.

Note. If $\lambda_{k}[\underline{\xi}]=\lambda_{k}[\underline{\eta}]$ for all $k$, then $[\underline{\xi}]=[\underline{\eta}]$. Let $\left(V, x_{0}\right)$ be a 
neighborhood of $\left(X, x_{0}\right)$ in $Q$. Choose $k$ so that $\left(X_{k}, x_{0}\right) \subset\left(V, x_{0}\right)$. Then $\lambda_{k}[\underline{\xi}]=\lambda_{k}[\underline{\eta}]$ implies there is an $m_{0}$ such that if $m \geqq m_{0}$ then $\xi_{m} \cong \eta_{m}$ in $\left(X_{k}, x_{0}\right) \subset\left(V, x_{0}\right)$.

We will now show that $\left(\pi_{n}^{Q}\left(X, x_{0}\right),\left\{\lambda_{k}\right\}\right)$ is a terminal object in the category $\operatorname{inv}\left(\pi_{n}(\underline{X})\right)$ [9], from which it will follow by uniqueness of inverse limit that $\underline{\pi}_{n}^{Q}\left(X, x_{0}\right) \approx \underline{\pi}_{n}\left(X, x_{0}\right)$. To show $\left(\underline{\pi}_{n}^{Q}\left(X, x_{0}\right),\left\{\lambda_{k}\right\}\right)$ is in the category inv $\left(\pi_{n}(\underline{X})\right)$, one must show that if $k \leqq k^{\prime}$ then $\lambda_{k}=\rho_{k k^{\prime}}, \lambda_{k}$, where $\rho_{k k^{\prime}}: \pi_{n}\left(X_{k^{\prime}}, x_{0}\right) \rightarrow \pi_{n}\left(X_{k}, x_{0}\right)$ is the homomorphism induced by $i_{k k^{\prime}}$ : $\left(X_{k^{\prime}}, x_{0}\right) \rightarrow\left(X_{k}, x_{0}\right)$. Choose $m_{0} \geqq m_{k}, m_{k^{\prime}}$. Then $\lambda_{k}[\underline{\xi}]=\left[\xi_{m_{0}}\right]=\rho_{k k^{\prime}} \lambda_{k}[\underline{\xi}]$.

It remains to show $\left(\underline{\pi}_{n}^{Q}\left(X, x_{0}\right),\left\{\lambda_{k}\right\}\right)$ is a terminal object; i.e., if $G$ is any group and $\sigma_{k}: G \rightarrow \pi_{n}\left(X_{k}, x_{0}\right)$ are group homomorphisms such that if $k \leqq k^{\prime}$ then $\sigma_{k}=\rho_{k k^{\prime}} \sigma_{k^{\prime}}$, then there is a unique group homomorphism $\sigma: G \rightarrow \underline{\pi}_{n}^{Q}\left(X, x_{0}\right)$ such that $\sigma_{k}=\lambda_{k} \sigma$ for all $k$. The uniqueness follows immediately from the above note.

Existence. Let $g \in G$. Define $\sigma(g)=\left[\left\{\xi_{k},(S, a) \rightarrow\left(X, x_{0}\right)\right\}\right]$ where $\xi_{k}:(S, a) \rightarrow\left(Q, x_{0}\right)$ satisfies $\xi_{k} \in \sigma_{k}(g) \in \pi_{n}\left(X_{k}, x_{0}\right)$. First, $\left\{\xi_{k},(S, a) \rightarrow\right.$ $\left.\left(X, x_{0}\right)\right\}$ is an approximative map of $(S, a)$ toward $\left(X, x_{0}\right)$. If $\left(U, x_{0}\right)$ is any neighborhood of $\left(X, x_{0}\right)$ in $Q$ choose $k_{0}$ such that $k \geqq k_{0}$ implies that $\left(X_{k}, x_{0}\right) \subset\left(U, x_{0}\right)$. Then $\sigma_{k}(g)=\rho_{k k+1} \sigma_{k+1}(g)$ so that $\xi_{k} \cong \xi_{k+1}$ in $\left(X_{k}, x_{0}\right) \subset\left(U, x_{0}\right)$. Next, $\sigma$ is a well-defined function for if $\xi=\left\{\xi_{k},(S, a) \rightarrow\right.$ $\left.\left(X, x_{0}\right)\right\}$ and $\underline{\xi}^{\prime}=\left\{\xi_{k}^{\prime},(S, a) \rightarrow\left(X, x_{0}\right)\right\}$ are such that $\xi_{k}, \xi_{k}^{\prime} \in \sigma_{k}(g)$ for each $k$, then if $\left(\bar{U}, x_{0}\right)$ is any neighborhood of $\left(X, x_{0}\right)$ in $Q$ choose $k_{0}$ such that if $k \geqq k_{0}$ then $\left(X_{k}, x_{0}\right) \subset\left(U, x_{0}\right)$. Then $\xi_{k} \cong \xi_{k}^{\prime}$ in $\left(U, x_{0}\right)$ and hence $[\xi]=\left[\xi^{\prime}\right]$. Also, $\sigma$ is a group homomorphism. Each $\sigma_{k}$ is a homomorphism so that $\sigma_{k}\left(g_{1} g_{2}\right)=\sigma_{k}\left(g_{1}\right) * \sigma_{k}\left(g_{2}\right)$. Thus if $\xi_{k} \in \sigma_{k}\left(g_{1}\right), \eta_{k} \in \sigma_{k}\left(g_{2}\right)$ then $\xi_{k} * \eta_{k} \in$ $\sigma_{k}\left(g_{1}\right) * \sigma_{k}\left(g_{2}\right)=\sigma_{k}\left(g_{1} g_{2}\right)$. That is, $\sigma\left(g_{1} g_{2}\right)=\left[\left\{\xi_{k} * \eta_{k},(S, a) \rightarrow\left(X, x_{0}\right)\right\}\right]$. But $\sigma\left(g_{1}\right) * \sigma\left(g_{2}\right)=\left[\left\{\xi_{k} * \eta_{k},(S, a) \rightarrow\left(X, x_{0}\right)\right\}\right]$ so that $\sigma$ is group homomorphism.

Finally, $\sigma_{k}=\lambda_{k} \sigma$ for each $k$. Since $\lambda_{k} \sigma(g)=\left[\xi_{m_{k}}\right] \in \pi_{n}\left(X_{k}, x_{0}\right)$, it suffices to show $\xi_{m_{k}} \cong \xi_{k}$ in $\left(X_{k}, x_{0}\right)$. If $k \geqq m_{k}$ then by the definition of $m_{k}, \xi_{k} \cong \xi_{m_{k}}$ in $\left(X_{k}, x_{0}\right)$. If $m_{k} \geqq k$ then $\sigma_{k}(g)=\rho_{k m_{k}} \sigma_{m_{k}}(g)$ so that $\xi_{m_{k}} \cong \xi_{k}$ in $\left(X_{k}, x_{0}\right)$.

This completes the proof of the theorem.

4. The product of a family of inverse systems. Let $\Omega$ be an index set. For each $\omega \in \Omega$, let $\underline{X}^{\omega}=\left\{X_{a}^{\omega}, p_{a a^{\prime}}^{\omega}, A^{\omega}\right\}$ be an inverse system of topological spaces (a similar construction can be made for groups, $R$-modules, etc.). Let $\Gamma=\{(F, \sigma): F$ is a finite nonempty subset of $\Omega$ and $\sigma: F \rightarrow \mathrm{U}_{\omega \in \Omega} A^{\omega}$ is a function such that $\sigma(\omega) \in A^{\omega}$ for all $\left.\omega \in F\right\}$. Order $\Gamma$ by $(F, \sigma) \leqq\left(F^{\prime \prime}, \sigma^{\prime}\right)$ iff $F \subset F^{\prime}$ and $\sigma(\omega) \leqq \sigma^{\prime}(\omega)$ for all $\omega \in F$. For $(F, \sigma) \in \Gamma$ let $X_{(F, \sigma)}=\prod_{\omega \in F} X_{\sigma(\omega)}^{\omega}$. If $(F, \sigma) \leqq\left(F^{\prime}, \sigma^{\prime}\right)$ then let $p_{(F, \sigma)\left(F^{\prime}, \sigma^{\prime}\right)}: X_{\left(F^{\prime} \sigma^{\prime}\right)} \rightarrow X_{(F, a)}$ be the composition of the natural projection

$$
\eta: \prod_{\omega \in F^{\prime}} X_{\sigma^{\prime}(\omega)}^{\omega} \longrightarrow \Pi_{\omega \in F} X_{\sigma^{\prime}(\omega)}^{\omega}
$$


and the product map $\Pi p_{v(\omega) \sigma^{\prime}(\omega)}^{\omega}: \prod_{\omega \in F} X_{\sigma^{\prime}(\omega)}^{\omega} \rightarrow \prod_{\omega \in F} X_{\sigma(\omega)}^{\omega}$. It is not difficult to show that $\underline{X}=\left\{X_{(F, \sigma)}, p_{\left(F^{\prime}, \sigma\right)\left(F^{\prime}, \sigma^{\prime}\right)}, \Gamma\right\}$ is an inverse system. The inverse system $\underline{X}$ is called the product of the family $\left\{\underline{X}^{\omega}: \omega \in \Omega\right\}$ and is denoted $\underline{X}=\Pi_{\omega \in \Omega} \underline{X}^{\omega}$. It can be shown that $\Pi_{\omega \in \Omega} \underline{X}^{\omega}$ is the categorical product of the family $\left\{\underline{X}^{\omega}: \omega \in \Omega\right\}$.

ExAMPLE. If each $A^{\omega}$ is a singleton, each $X_{a}^{\omega}=I_{\omega}=I$ is the unit interval and $\underline{X}^{\omega}=\left\{I_{\omega}, 1_{\omega}\right\}$ where $1_{\omega}: I_{\omega} \rightarrow I_{\omega}$ is the identity map then the above construction gives the usual representation of $I^{\Omega}=\prod_{\omega \in \Omega} I_{\omega}$ as the inverse limit of $\left\{I^{\alpha}, p_{\alpha \alpha^{\prime}}, F(\Omega)\right\}$ where $F(\Omega)$ is the set of all nonempty finite subsets of $\Omega$ ordered by inclusion and $p_{\alpha \alpha^{\prime}}: I^{\alpha^{\prime}}=\Pi_{\omega \in \alpha^{\prime}}$ $I_{\omega} \rightarrow I^{\alpha}$, is the natural projection (see [6]).

\section{THeOREM 4.1. $\lim _{\omega \in \Omega} \underline{X}^{\omega}=\prod_{\omega \in \Omega} \stackrel{\lim }{\underline{X}^{\omega}}$.}

Proof. Let $X^{\omega}=\lim \underline{X}^{\omega}$. We show $\Pi_{\omega \in \Omega} X^{\omega}$ is a terminal object in the category $\operatorname{inv}\left(\prod_{\omega \in \Omega} \underline{X}^{\omega}\right)$. For $(F, \sigma) \in \Gamma$, let $p_{(F, \sigma)}: \prod_{\omega \in \Omega} X^{\omega} \rightarrow$ $X_{(F, o)}$ be the composition of the natural projection $\eta: \prod_{\omega \in \Omega} X^{\omega} \rightarrow \Pi_{\omega \in F} X^{\omega}$, and the product map $\Pi p_{\sigma(\omega)}^{\omega}: \prod_{\omega \in F} X^{\omega} \rightarrow \prod_{\omega \in F} X_{\sigma(\omega)}^{\omega}$. It is not hard to show that if $(F, \sigma) \leqq\left(F^{\prime}, \sigma^{\prime}\right)$ then $p_{(F, \sigma)\left(F^{\prime}, \sigma^{\prime}\right)} p_{\left(F^{\prime}, \sigma^{\prime}\right)}=p_{(F, o)}$. Thus $\left(\Pi_{\omega \in \Omega} X^{\omega},\left\{p_{(F, \sigma)}\right\}\right)$ is in the category $\operatorname{inv}\left(\Pi \underline{X}^{\omega}\right)$.

It remains to show that $\prod_{\omega \in \Omega} X^{\omega}$ is a terminal object. That is, if $Y$ is any space and $f_{(F, o)}: Y \rightarrow X_{(F, a)}$ is a family of maps such that if $(F, \sigma) \leqq\left(F^{\prime}, \sigma^{\prime}\right)$ then $p_{(F, \sigma)\left(F^{\prime}, \sigma^{\prime}\right)} f_{\left(F^{\prime}, \sigma^{\prime}\right)}=f_{(F, \sigma)}$, then there is a unique map $f: Y \rightarrow \prod_{\omega \in \Omega} X^{\omega}$ such that for all $(F, \sigma) \in \Gamma, p_{(F, \sigma)} f=f_{(F, \sigma)}$. If $\omega \in \Omega$ and $a \in A^{\omega}$ let $\sigma_{a}:\{\omega\} \rightarrow A^{\omega}$ be the function $\sigma_{a}(\omega)=a$. Then $\left(\{\omega\}, \sigma_{a}\right) \in \Gamma$ and $f_{a}^{\omega}=f_{\left(\{\omega\}, \sigma_{a}\right)}: Y \rightarrow X_{a}^{\omega}$ is a family of maps such that if $a \leqq a^{\prime}$ then $\left(\{\omega\}, \sigma_{a}\right) \leqq\left(\{\omega\}, \sigma_{a^{\prime}}\right)$, so that $p_{a a^{\prime}}^{\omega} f_{a^{\prime}}^{\omega}=f_{a}^{\omega}$. By the universal mapping property of $X^{\omega}$, there is a unique $f^{\omega}: Y \rightarrow X^{\omega}$ such that $p_{a}^{\omega} f^{\omega}=f_{a}^{\omega}$ for all $a \in A^{\omega}$. Let $f: Y \rightarrow \prod_{\omega \in \Omega} X^{\omega}$ be the unique map thus defined. Then $f$ satisfies $p_{(F, o)} f=f_{(F, \sigma)}$. Furthermore, if $g: Y \rightarrow$ $\Pi_{\omega \in \Omega} X^{\omega}$ is any map that satisfies $p_{(F, \sigma)} g=f_{(F, \sigma)}$ then $p_{\left(\{\omega\}, \sigma_{a}\right)} g=f_{\left(\{\omega\}, \sigma_{a}\right)}=$ $f_{a}^{\omega}$. It follows then that $f=g$.

CoROLlary 4.2. If $X^{\omega}=\left\{X_{a}^{\omega}, p_{a a^{\prime}}^{\omega}, A^{\omega}\right\}, \omega \in \Omega$, is a family of $A N R$ systems where $\underline{X}^{\omega}$ is associated with $X^{\omega}$, then $\Pi_{\omega \in \Omega} \underline{X}^{\omega}$ is an ANRsystem associated with $\Pi_{\omega \in \Omega} X^{\omega}$.

Proof. It suffices to note that if each $A^{\omega}$ is closure-finite then so is $\Gamma$ and that the product of a finite number of ANR's is an ANR.

Suppose $\underline{X}^{\omega}=\left\{X_{a}^{\omega}, p_{a a^{\prime}}^{\omega}, A^{\omega}\right\}, \omega \in \Omega ; \underline{Y}^{\lambda}=\left\{Y_{b}^{\lambda}, q_{b b^{\prime}}^{\lambda}, B^{\lambda}\right\}, \lambda \in \Lambda$, are inverse systems (or ANR-systems) and $\theta: \bar{\Lambda} \rightarrow \Omega$ is a one-to-one function such that for each $\lambda \in \Lambda$ there is a map $\underline{f}^{\lambda}: \underline{X}^{\theta(\lambda)} \rightarrow \underline{Y}^{\lambda}$. Recall, a map 
$\underline{f}^{\lambda}: \underline{X}^{\theta(\lambda)} \rightarrow \underline{Y}^{\lambda}$ consists of an increasing function $f^{\lambda}: B^{\lambda} \rightarrow A^{\theta(\lambda)}$ together with a family of maps $f_{b}^{\lambda}: X_{f^{\lambda}(b)}^{\lambda^{(\lambda)}} \rightarrow Y_{b}^{\lambda}, b \in B^{\lambda}$, such that if $b \leqq b^{\prime}$ then $q_{b b^{\prime}}^{\lambda} f_{b^{\prime}}^{\lambda}=f_{b}^{\lambda}$ (in the ANR-system case, $q_{b b^{\prime}}^{\lambda} f_{b^{\prime}}^{\lambda} \cong f_{b}^{\lambda}$ ). Define $f: \Gamma_{Y} \rightarrow \Gamma_{X}$ by $f(F, \sigma)=\left(\theta(F), f_{\sigma}\right)$ where $f_{\sigma}: \theta(F) \rightarrow \bigcup_{\omega \in \Omega} A^{\omega}$ is given by $f_{\sigma}(\theta(\lambda))=$ $f^{\lambda}(\sigma(\lambda)) \in A^{\theta(\lambda)}$. Then $X_{f(F, \sigma)}=\prod_{\omega \in \theta(F)} X_{f_{\sigma}(\omega)}^{\omega}=\prod_{\omega \in F} X_{\left.f^{\prime}(\alpha)(\lambda)\right)}^{\lambda^{(\lambda)}}$ so define $f_{(F, \sigma)}: X_{f(F, \sigma)} \rightarrow Y_{(F, \sigma)}$ as the product map $\Pi f_{\sigma(\lambda)}^{\lambda}: \prod_{\lambda \in F} X_{f^{\lambda}(\sigma(\lambda))}^{\theta^{(\lambda)}} \rightarrow \prod_{\lambda \in F} Y_{\sigma(\lambda)}^{\lambda}$. One then checks that if $(F, \sigma) \leqq\left(F^{\prime \prime}, \sigma^{\prime}\right)$ then $f(F, \sigma) \leqq f\left(F^{\prime \prime}, \sigma^{\prime}\right)$ and $q_{(F, \sigma)\left(F^{\prime}, \sigma^{\prime}\right)} f_{\left\langle F^{\prime}, \sigma^{\prime}\right)}=f_{(F, \sigma)}$ (in the ANR-system case, $\left.q_{(F, \sigma)\left\langle F^{\prime}, \sigma^{\prime}\right)} f_{\left(F^{\prime}, \sigma^{\prime}\right)} \cong f_{(F, \sigma)}\right)$. Thus there is a map $f: \prod_{\omega \in \Omega} \underline{X}^{\omega} \rightarrow \prod_{\lambda \in \Lambda} \underline{Y}^{\lambda}$.

If $\underline{Z}^{\tau}=\left\{Z_{c}^{-}, r_{c c^{\prime}}^{\tau}, \bar{C}^{\tau}\right\}, \tau \in T$, is another family of inverse systems and $\phi: \Omega \rightarrow T$ is a one-to-one function such that for all $\omega \in \Omega$ there is a $\underline{g}^{\omega}: \underline{Z}^{\phi(\omega)} \rightarrow \underline{X}^{\omega}$ then there is a "natural composition" given by $\phi \theta: \Lambda \rightarrow$ $T$ and $\underline{f}^{\lambda} \underline{g}^{\theta(\lambda)}: \underline{Z}^{\dot{\theta}(\lambda)} \rightarrow \underline{Y}^{\lambda}$. It is left to the reader to verify that the map determined by the composition is the same as the composition of the respective determined maps.

There is a "natural identity", $\theta: \Omega \rightarrow \Omega$ the identity function and each $\underline{1}^{\omega}: \underline{X}^{\omega} \rightarrow \underline{X}_{\omega}$ the identity map. It is left to the reader to verify that the identity $1: \prod_{\omega \in \Omega} \underline{X}^{\omega} \rightarrow \prod_{\omega \in \Omega} \underline{X}^{\omega}$ is determined by the natural identity.

We now restrict our attention to the ANR-system case when $Q=$ $A$ and $\theta$ is the identity.

THeOREM 4.3. If $\underline{f}^{\omega}, \underline{g}^{\omega}: \underline{X}^{\omega} \rightarrow \underline{Y}^{\omega}$ are families of maps of systems such that $\underline{f}^{\omega} \cong \underline{g}^{\omega}$ for all $\omega \in \Omega$ then $\underline{f} \cong \underline{g}$ : $\prod_{\omega \in \Omega} \underline{X}^{\omega} \rightarrow \prod_{\omega \in \Omega} \underline{Y}^{\omega}$.

Proof. For each $b \in B^{\omega}$ there is an $a_{b} \in A^{\omega}, a_{b} \geqq f^{\omega}(b), g^{\omega}(b)$ such that $f_{b}^{\omega} p_{f^{\omega}(b) a_{b}}^{\omega^{\omega}} \cong g_{b}^{\omega} p_{g^{\omega}(b) a_{b}}^{\omega}$. Let $\tau_{\omega}: B^{\omega} \rightarrow A^{\omega}$ be an increasing function such that $\tau_{\omega}(b) \geqq a_{b}$ for all $b \in B^{\omega}$. If $(F, \sigma) \in \Gamma_{Y}$, consider $(F, \tau) \in \Gamma_{X}$ where $\tau: F \rightarrow \bigcup_{\omega \in \Omega} A^{\omega}$ is given by $\tau(\omega)=\tau_{\omega}(\sigma(\omega))$. First, $(F, \tau) \geqq$ $f(F, \sigma), g(F, \sigma)$. Since $\theta$ is the identity, $f(F, \sigma)=\left(F, f_{\sigma}\right)$ where $f_{\sigma}(\omega)=$ $f^{\omega}(\sigma(\omega))$. Then $(F, \tau) \geqq\left(F, f_{\sigma}\right)$ since

$$
\tau(\omega)=\tau_{\omega}(\sigma(\omega)) \geqq a_{\sigma(\omega)} \geqq f^{\omega}(\sigma(\omega))=f_{\sigma}(\omega) .
$$

Similarly, $(F, \sigma) \geqq g(F, \sigma)$. Furthermore,

$$
f_{\sigma(\omega)}^{\omega} p_{f^{\omega}(\sigma(\omega)) \tau_{\omega^{\omega}}(\sigma(\omega))}^{\omega} \cong g_{\sigma(\omega)}^{(\omega)} p_{g^{(\omega}(\sigma(\omega)) \tau_{\omega}(\sigma(\omega))}^{\omega}
$$

implies

$$
f_{(F, \sigma)} p_{f(F, \sigma)(F,-)} \cong g_{(F, \sigma)} p_{g(F, \sigma)(F, \tau)} \cdot
$$

Thus $\underline{f} \cong \underline{g}$. 


$$
\operatorname{Sh}\left(\Pi_{\omega \in \Omega} X^{\omega}\right)=\operatorname{Sh}\left(\Pi_{\omega \in \Omega} Y^{\omega}\right) .
$$

Corollary 4.4 allows one to define the product of shapes as follows: $\Pi_{\omega \in \Omega} \operatorname{Sh}\left(X^{\omega}\right)=\operatorname{Sh}\left(\prod_{\omega \in \Omega} X^{\omega}\right)$.

In [5] Mardešic gives the notion of a shape retraction. For our purposes we use the following definition: if $j: X \rightarrow Y$ is an embedding then a map of systems $\underline{r}: \underline{Y} \rightarrow \underline{X}$ is a shape retraction iff $\underline{r j} \cong \underline{1}_{\underline{X}}$ where $\underline{j}: \underline{X} \rightarrow \underline{Y}$ is a map of systems associated with $j$. It is routine to verify this definition is equivalent to the one given by Mardešić. If there is an embedding $j: X \rightarrow Y$ and a shape retraction $\underline{r}: \underline{Y} \rightarrow \underline{X}$ then $X$ is said to be a shape retract of $Y$.

COROLLARY 4.5. If $\underline{r}^{\omega}: \underline{Y}^{\omega} \rightarrow \underline{X}^{\omega}$ is a shape retraction for all $\omega$ then $\underline{r}: \Pi_{\omega \in \Omega} \underline{Y}^{\omega} \rightarrow \Pi_{\omega \in \Omega} \underline{X}^{\omega}$ is also a shape retraction.

Proof. Let $\underline{Y}^{\omega}, \underline{X}^{\omega}$ be associated with $Y^{\omega}, X^{\omega}$, respectively, and $j^{\omega}: X^{\omega} \rightarrow Y^{\omega}$ the required embeddings. Let $j: \prod_{\omega \in \Omega} X^{\omega} \rightarrow \prod_{\omega \in \Omega} Y^{\omega}$ be the embedding determined by the family $\left\{j^{\omega}: \omega \in \Omega\right\}$. It is routine to verify that the map determined by the family $\left\{j^{\omega}: \underline{X}^{\omega} \rightarrow \underline{Y}^{\omega}\right\}$ is associated with $j$. We have that $\underline{r}^{\omega} \underline{j}^{\omega} \cong \underline{1}_{\omega}$ where $\underline{1}_{\omega}: \underline{X}^{\omega} \rightarrow \underline{X}^{\omega}$ is the map associated with the identity. By the above theorem, $\underline{r j} \cong \underline{1}_{\Pi_{\omega \in \Omega} \underline{X} \omega}$.

5. Products of ASR and ANSR-sets. In [5] Mardešić gives definitions for absolute shape retract (ASR) and absolute neighborhood shape retracts (ANSR). These correspond to Borsuk's FAR and FANRsets, respectively, in the metric case. We will use the following characterizations: A compact Hausdorff space $X$ is an ASR (respectively, ANSR) if there is a compact AR (respectively, ANR) $Y$ and an embedding $j: X \rightarrow Y$ such that $X$ is a shape retract of $Y$ (see [10] and [5]).

THEOREM 5.1. If $X=\prod_{\omega \in \Omega} X^{\omega}$ then $X \in A S R$ iff $X^{\omega} \in A S R$ for all $\omega \in \Omega$.

Proof. If $X \in \mathrm{ASR}$ there is a $Y \in \mathrm{AR}$, an embedding $j: X \rightarrow Y$ and a shape retraction $\underline{r}: \underline{Y} \rightarrow \underline{X}$. Since each natural projection $p_{\omega}: X \rightarrow$ $X^{\omega}$ is a retraction, the associated maps of systems $\underline{p}_{\omega}: \underline{X} \rightarrow \underline{X}^{\omega}$ are shape retractions. It follows [5] that $\underline{p_{\omega}} \underline{r}: \underline{Y} \rightarrow \underline{X}^{\omega}$ is a shape retraction. Thus, each $X^{\omega}$ is an ASR.

Conversely, if $X^{\omega} \in$ ASR for all $\omega \in \Omega$, then for each $\omega \in \Omega$ there is an $\mathrm{AR}$-set $Y^{\omega}$ such that $\underline{X}^{\omega}$ is a shape retract of $\underline{Y}^{\omega}$. Since the product of any family of AR-sets is an AR-set, we have by Corollary 4.5 that $X \in$ ASR. 
THEOREM 5.2. If $X=\prod_{\omega \in \Omega} X^{\omega}$ then $X \in A N S R$ iff $X^{\omega} \in A N S R$ for all $\omega$ and $X^{\omega} \in A S R$ for all but a finite number of $\omega$.

Proof. If $X^{\omega} \in$ ANSR for all $\omega$ and $X^{\omega} \in$ ASR for all but finitely many $\omega$, say $\omega_{1}, \omega_{2}, \cdots, \omega_{n}$, then for all $\omega$ there is an ANR-set $Y^{\omega}$ and a shape retraction $\underline{r}^{\omega}: \underline{Y}^{\omega} \rightarrow \underline{X}^{\omega}$ such that $Y^{\omega} \in \mathrm{AR}$ if $\omega \neq \omega_{k}(k=$ $1,2, \cdots, n)$. Then $\prod_{\omega \in \Omega} Y^{\omega} \in$ ANR and there is a shape retraction $\underline{r}: \prod_{\omega \in \Omega} \underline{Y}^{\omega} \rightarrow \Pi_{w \in \Omega} \underline{X}^{\omega}$ so that $\prod_{\omega \in \Omega} X^{\omega} \in$ ANSR.

Conversely, if $X \in$ ANSR then as in the proof of Theorem 5.1, each $X^{\omega} \in$ ANSR. We may assume without loss that $X^{\omega} \subset I^{\Lambda \omega}=\Pi_{\lambda \in \Lambda \omega} I^{\lambda}$ and $X \subset I^{A}=\prod_{\omega \in \Omega} I^{A \omega}$. By Theorem IV. 2.10 of [10], there is a closed neighborhood $W$ of $X$ in $I^{4}$ and a shape retraction $\underline{r}: \underline{W} \rightarrow \underline{X}$. There is a finite subset of $\Omega,\left\{\omega_{1}, \omega_{2}, \cdots, \omega_{n}\right\}$ and neighborhoods $U_{i}$ of $X^{\omega_{i}}$ in $I^{\Lambda_{\omega_{i}}}(i=1,2, \cdots, n)$ such that

$$
X=\prod_{\omega \in \Omega} X^{\omega} \subset \prod_{i=1}^{n} U_{i} \times \prod_{\omega \neq \omega_{i}} I^{\Lambda_{\omega}} \subset W .
$$

Let $i: X \rightarrow W, j_{\omega}: X^{\omega} \rightarrow I^{\Lambda_{\omega}}$ denote the inclusion maps and let $p_{\omega}: X \rightarrow$ $X^{\omega}$ be the natural projections. Choose inclusion maps $j_{\omega}^{\prime}: I^{A}{ }_{\omega} \rightarrow W$ for $\omega \neq \omega_{i}(i=1,2, \cdots, n)$ and $i_{\omega}: X^{\omega} \rightarrow X$ such that $j_{\omega}^{\prime} j_{\omega}=i i_{\omega}$ and $p_{\omega} i_{\omega}=$ $1_{X_{\omega}}$. Then $\underline{r i} \cong \underline{1}_{\underline{X}}$ so that for $\omega \neq \omega_{i}(i=1,2, \cdots, n)$,

$$
\underline{p}_{\omega} \underline{r j}_{-}^{\prime} \underline{j}_{\omega} \cong \underline{p}_{\omega} \underline{r i i_{\omega}} \cong \underline{p}_{\omega} \underline{i}_{\omega} \cong \underline{1}_{x} \omega .
$$

Hence $\underline{p}_{\omega} \underline{\underline{r}}_{\omega}^{\prime}: \underline{I}^{\Lambda_{\omega}} \rightarrow \underline{X}^{\omega}$ is a shape retraction for $\omega \neq \omega_{i}(i=1,2, \cdots, n)$. Thus $X^{\omega}, \omega \neq \omega_{i}(i=1,2, \cdots, n)$, is an ASR-set.

6. Products and shape groups. An inspection of Theorem 4.1 shows that the proof does not involve the fact that each $X^{\omega}$ is a topological space. It remains valid, for example, whenever the objects are groups. This fact together with the fact that the (usual) homotopy group of a product is the direct product of the (usual) homotopy groups of its factors, [11] Exercise B.5, p. 419, gives the following theorem.

THEOREM 6.1. If $\left(X, x_{0}\right)=\prod_{\omega \in \Omega}\left(X^{\omega}, x_{0}^{\omega}\right)$ then $\underline{\pi}_{n}\left(X, x_{0}\right)=$ $\Pi_{\omega \in \Omega} \underline{\pi}_{n} \quad\left(X^{\omega}, x_{0}^{\omega}\right)$.

Proof. For each $\omega$ let $\underline{X}^{\omega}=\left\{\left(X_{a}^{\omega}, x_{a}^{\omega}\right), p_{a a^{\prime}}^{\omega}, A^{\omega}\right\}$ be an ANR-system associated with $\left(X^{\omega}, x_{0}^{\omega}\right)$. Then

$$
\begin{aligned}
\underline{\pi}_{n}\left(X, x_{0}\right) & =\underline{\pi}_{n}\left(\prod_{\omega \in \Omega}\left(X^{\omega}, x_{0}^{\omega}\right)\right) \\
& =\underline{\lim } \pi_{n}\left(\prod_{\omega \in \Omega} \underline{X}^{\omega}\right)
\end{aligned}
$$




$$
\begin{aligned}
& =\lim _{n} \pi_{n}\left\{X_{(F, \sigma)}, p_{(F, \sigma)\left(F^{\prime}, \sigma^{\prime}\right)}, \Gamma\right\} \\
& =\lim _{\longleftarrow}\left\{\pi_{n}\left(\prod_{\omega \in F}\left(X_{\sigma(\omega)}^{\omega}, x_{\sigma(\omega)}^{\omega}\right), \rho_{(F, \sigma)\left(F^{\prime}, \sigma^{\prime}\right)}, \Gamma\right\}\right. \\
& =\lim _{\longleftarrow}\left\{\prod_{\omega \in F} \pi_{n}\left(X_{\sigma(\omega)}^{\omega}, x_{\sigma(\omega)}^{\omega}\right), \prod_{\omega \in F} \rho_{\sigma(\omega) \sigma^{\prime}(\omega)} \eta, \Gamma\right\} \\
& =\lim _{\omega \in \Omega} \prod_{\omega}\left\{\pi_{n}\left(X_{a}^{\omega}, x_{a}^{\omega}\right), \rho_{a a^{\prime}}^{\omega}, A^{\omega}\right\} \\
& =\prod_{\omega \in \Omega} \lim _{\longleftarrow}\left\{\pi_{n}\left(X_{a}^{\omega}, x_{a}^{\omega}\right), \rho_{a a^{\prime}}^{\omega}, A^{\omega}\right\} \\
& =\prod_{\omega \in \Omega} \underline{\pi}_{n}\left(X^{\omega}, x_{0}^{\omega}\right) \text {. }
\end{aligned}
$$

Note. In recent correspondence M. Moszyńska indicated that she has defined the concept of "limit homotopy groups" which correspond to our definition of the shape groups. Her approach, to appear in [8] and [9], is more categorical than ours. For completeness we have included our definition and proof that the shape are isomorphic to Borsuk's fundamental groups. The approach to the latter (Theorem 3.1) is somewhat different than the approach she used (see [9]).

\section{REFERENCES}

1. K. Borsuk, Concerning homotopy properties of compacta, Fund. Math., 62 (1968), 223-254.

2. - Fundamental retracts and extensions of fundamental sequences, Fund. Math., 64 (1969), 55-85.

3. — On the concept of shape for metrizable spaces, Bull. Acad. Polon. Sci. Ser. Sci. Math. Astr. Phys., 18 (1970), 127-132.

4. - Some remarks concerning the shape of pointed compacta, Fund. Math., 67 (1970), 221-240.

5. S. Mardešić, Retracts in Shape Theory, Glasnik Mathematički, 6 (1971), 153-163.

6. S. Mardešić and J. Segal, Shapes of compacta and ANR-systems, Fund. Math., 72 (1971), 41-59.

7. Equivalence of the Borsuk and the ANR-system Approach to Shapes, Fund. Math., 72 (1971), 61-68.

8. M. Moszyńska, Uniformly movable compact spaces and their algebraic properties, Fund. Math., 77, 2.

9. - Various approaches to the fundamental groups, Fund. Math., 78, 2.

10. T. Sanders, Shape Groups, ANR-systems and Related Topics, Ph. D. Dissertation, The University of Oklahoma, 1972.

11. E. H, Spanier, Algebraic Topology, McGraw-Hill, 1966.

Received June 23, 1972 and in revised form August 3, 1972. This paper is taken from the author's Ph. D. dissertation which was done under the direction of Dr. Leonard Rubin at The University of Oklahoma, Norman, Oklahoma.

UNIVERSITY OF OKLAHOMA

AND

Virginia Polytechnic Institute and State University

Current address: Department of Mathematics

U. S. Naval Academy

Annapolis, Maryland 21402 


\section{PACIFIC JOURNAL OF MATHEMATICS}

\section{EDITORS}

RICHARD ARENS (Managing Editor)

University of California

Los Angeles, California 90024

\author{
R. A. Beaumont \\ University of Washington \\ Seattle, Washington 98105
}

J. Dugundu*

Department of Mathematics

University of Southern California

Los Angeles, California 90007

D. Gilbarg and J. Milgram

Stanford University

Stanford, California 94305

\section{ASSOCIATE EDITORS}
E. F. BECKENBACH
B. H. NeumanN
F. WOLF
K. YosHIDA

\section{SUPPORTING INSTITUTIONS}

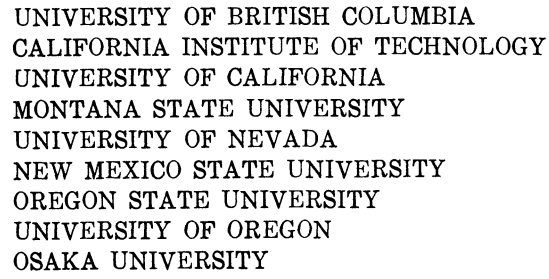

UNIVERSITY OF BRITISH COLUMBIA CALIFORNIA INSTITUTE OF TECHNOLOGY

UNIVERSITY OF CALIFORNIA

MONTANA STATE UNIVERSITY

UNIVERSITY OF NEVADA

NEW MEXICO STATE UNIVERSITY

OREGON STATE UNIVERSITY

UNIVERSITY OF OREGON

OSAKA UNIVERSITY

\author{
UNIVERSITY OF SOUTHERN CALIFORNIA \\ STANFORD UNIVERSITY \\ UNIVERSITY OF TOKYO \\ UNIVERSITY OF UTAH \\ WASHINGTON STATE UNIVERSITY \\ UNIVERSITY OF WASHINGTON \\ * * * \\ AMERICAN MATHEMATICAL SOCIETY \\ NAVAL WEAPONS CENTER
}

The Supporting Institutions listed above contribute to the cost of publication of this Journal, but they are not owners or publishers and have no responsibility for its content or policies.

Mathematical papers intended for publication in the Pacific Journal of Mathematics should be in typed form or offset-reproduced, (not dittoed), double spaced with large margins. Underline Greek letters in red, German in green, and script in blue. The first paragraph or two must be capable of being used separately as a synopsis of the entire paper. Items of the bibliography should not be cited there unless absolutely necessary, in which case they must be identified by author and Journal, rather than by item number. Manuscripts, in duplicate if possible, may be sent to any one of the four editors. Please classify according to the scheme of Math. Rev. Index to Vol. 39. All other communications to the editors should be addressed to the managing editor, or Elaine Barth, University of California, Los Angeles, California, 90024.

50 reprints are provided free for each article; additional copies may be obtained at cost in multiples of 50 .

The Pacific Journal of Mathematics is issued monthly as of January 1966. Regular subscription rate: $\$ 48.00$ a year (6 Vols., 12 issues). Special rate: $\$ 24.00$ a year to individual members of supporting institutions.

Subscriptions, orders for back numbers, and changes of address should be sent to Pacific Journal of Mathematics, 103 Highland Boulevard, Berkeley, California, 94708.

PUBLISHED BY PACIFIC JOURNAL OF MATHEMATICS, A NON-PROFIT CORPORATION

Printed at Kokusai Bunken Insatsusha (International Academic Printing Co., Ltd.), 270, 3-chome Totsuka-cho. Shinjuku-ku, Tokyo 160, Japan.

* C. R. DePrima California Institute of Technology, Pasadena, CA 91109, will replace J. Dugundji until August 1974.

Copyright (C) 1973 by

Pacific Journal of Mathematics

All Rights Reserved 


\section{Pacific Journal of Mathematics}

Vol. 48, No. $2 \quad$ April, 1973

Mir Maswood Ali, Content of the frustum of a simplex................

Mieczyslaw Altman, Contractors, approximate identities and factorization

in Banach algebras ................................ 323

Charles Francis Amelin, A numerical range for two linear operators ...... 335

John Robert Baxter and Rafael Van Severen Chacon, Nonlinear functionals

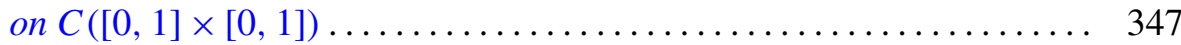

Stephen Dale Bronn, Cotorsion theories....................... 355

Peter A. Fowler, Capacity theory in Banach spaces............... 365

Jerome A. Goldstein, Groups of isometries on Orlicz spaces ........... 387

Kenneth R. Goodearl, Idealizers and nonsingular rings . ............ 395

Robert L. Griess, Jr., Automorphisms of extra special groups and

nonvanishing degree 2 cohomology ..................... 403

Paul M. Krajkiewicz, The Picard theorem for multianalytic functions . . . . 423

Peter A. McCoy, Value distribution of linear combinations of axisymmetric harmonic polynomials and their derivatives ...................

A. P. Morse and Donald Chesley Pfaff, Separative relations for

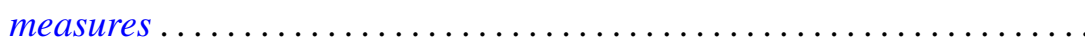

Albert David Polimeni, Groups in which $\operatorname{Aut}(G)$ is transitive on the

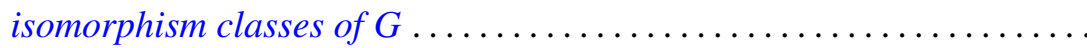

Aribindi Satyanarayan Rao, Matrix summability of a class of derived

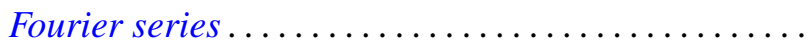

Thomas Jay Sanders, Shape groups and products

Ruth Silverman, Decomposition of plane convex sets. II. Sets associated

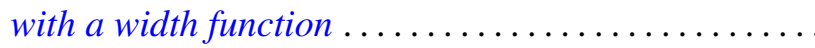

Richard Snay, Decompositions of $E^{3}$ into points and countably many flexible dendrites.............................

John Griggs Thompson, Nonsolvable finite groups all of whose local subgroups are solvable, IV ...

Robert E. Waterman, Invariant subspaces, similarity and isometric equivalence of certain commuting operators in $L_{p} \ldots$

James Chin-Sze Wong, An ergodic property of locally compact amenable

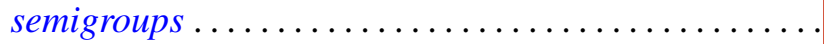

Julius Martin Zelmanowitz, Orders in simple Artinian rings are strongly equivalent to matrix rings ....................... 